\title{
In Silico QT and APD Prolongation Assay for Early Screening of Drug- Induced Proarrhythmic Risk
}

\author{
Lucia Romero, ${ }^{\dagger, \S}$ Jordi Cano, ${ }^{\dagger, \S ®}$ Julio Gomis-Tena, ${ }^{\dagger}$ Beatriz Trenor, ${ }^{\dagger}$ Ferran Sanz, ${ }^{\ddagger}$ Manuel Pastor, ${ }^{\ddagger}$ \\ and Javier Saiz ${ }^{* \dagger}$
}

${ }^{\dagger}$ Centro de Investigación e Innovación en Bioingeniería $\left(\mathrm{CI}^{2} \mathrm{~B}\right)$, Universitat Politècnica de València, camino de Vera, s/n, 46022 Valencia, Spain

${ }^{*}$ Research Programme on Biomedical Informatics (GRIB), Institut Hospital del Mar d'Investigacions Mèdiques (IMIM), Department of Experimental and Health Sciences, Universitat Pompeu Fabra, Carrer del Dr. Aiguader 88, 08002 Barcelona, Spain

\section{Supporting Information}

\begin{abstract}
Drug-induced proarrhythmicity is a major concern for regulators and pharmaceutical companies. For novel drug candidates, the standard assessment involves the evaluation of the potassium hERG channels block and the in vivo prolongation of the QT interval. However, this method is known to be too restrictive and to stop the development of potentially valuable therapeutic drugs. The aim of this work is to create an in silico tool for early detection of drug-induced proarrhythmic risk. The system is based on simulations of how different compounds affect the action potential duration

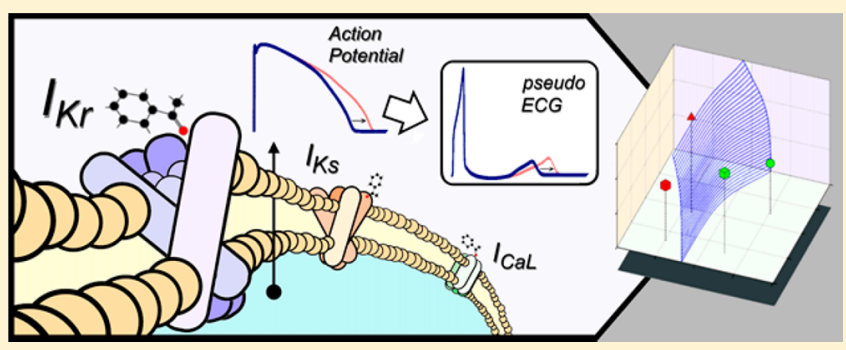
(APD) of isolated endocardial, midmyocardial, and epicardial cells as well as the QT prolongation in a virtual tissue. Multiple channel-drug interactions and state-of-the-art human ventricular action potential models (O'Hara, T., et al. PLos Comput. Biol. 2011, 7, e1002061) were used in our simulations. Specifically, 206.766 cellular and 7072 tissue simulations were performed by blocking the slow and the fast components of the delayed rectifier current ( $I_{\mathrm{Ks}}$ and $I_{\mathrm{Kr}}$, respectively) and the L-type calcium current $\left(I_{\mathrm{CaL}}\right)$ at different levels. The performance of our system was validated by classifying the proarrhythmic risk of 84 compounds, 40 of which present torsadogenic properties. On the basis of these results, we propose the use of a new index (Tx) for discriminating torsadogenic compounds, defined as the ratio of the drug concentrations producing $10 \%$ prolongation of the cellular endocardial, midmyocardial, and epicardial APDs and the QT interval, over the maximum effective free therapeutic plasma concentration (EFTPC). Our results show that the Tx index outperforms standard methods for early identification of torsadogenic compounds. Indeed, for the analyzed compounds, the Tx tests accuracy was in the range of $87-88 \%$ compared with a $73 \%$ accuracy of the hERG IC $_{50}$ based test.
\end{abstract}

\section{INTRODUCTION}

Preclinical assessment of cardiac adverse effects for drug candidates has become a priority for regulatory agencies and pharmaceutical companies due to the occurrence of potentially life-threatening ventricular arrhythmias following the administration of drugs. ${ }^{1-5}$ Nowadays, cardiac safety tests of pharmacological compounds are mainly based on the observed in vitro block of the human ether-à-go-go-related gene, hERG, current (encoding the pore-forming $\alpha$-subunit of the rapid component of delayed rectifier current $\left(I_{\mathrm{Kr}}\right)$ channels in humans) produced by the compound, and the in vivo prolongation of the QT interval ( $\mathrm{ICH}$ S7B nonclinical guidance $^{6}$ and E14 clinical guidance ${ }^{7}$ ).

Indeed, many studies have revealed that block of $I_{\mathrm{Kr}}$ promotes the drug-induced long QT syndrome, associated with increased proarrhythmic risk. ${ }^{8}$ Prolongation of the QT interval has traditionally been linked to the initiation of torsade de pointes $(\mathrm{TdP})$ in the clinical practice. However, there are hERG blockers that do not lead to TdP such as verapamil., ${ }^{9,10}$
Although current guidelines ${ }^{6,7}$ have been successful in preventing proarrhythmic effects of drugs, the limitations of these criteria have contributed to stop the development of potentially valuable compounds. A more accurate prediction of unwanted effects of drug candidates at early stages of drug development is key for safety pharmacology. ${ }^{11}$

In the last years, different modeling techniques have been employed in order to improve the prediction of side effects of compounds. Biophysically detailed mathematical models of cardiac electrical activity ${ }^{12-16}$ and statistical models ${ }^{9,17-19}$ have been used in these studies. Additionally, the work of Lancaster and Sobie ${ }^{20}$ combines both types of models. A nice comparison of different classification schemes can be found in a recent review of Wisniowska and Polak. ${ }^{21}$ The use of modeling and simulation tools of the cardiac electrophysiological activity to predict the proarrhythmic risk of drugs is currently being

Received: July 21, 2017

Published: March 16, 2018 
Table 1. Characteristics of the 84 Drugs Used in This Study: Name (First Column), Torsadogenic Risk (Second Column and Color-Coded Background of the First Column: Red (Class 1), Orange (Class 2), Bright-Green (Class 3 ) and Dark-Green (Class 4)), $\mathrm{pIC}_{50}\left(-\log \mathrm{IC}_{50}\right)$ Values for hERG, $I_{\mathrm{Ks}}$, and $I_{\mathrm{CaL}}$ (Third to Fifth column) and the EFTPC (Sixth Column) ${ }^{a}$

\begin{tabular}{|c|c|c|c|c|c|c|c|c|c|c|c|}
\hline \multirow[b]{2}{*}{ Compound Name } & \multirow[b]{2}{*}{ Class } & \multicolumn{3}{|c|}{$\mathrm{pIC}_{50}$} & \multirow[b]{2}{*}{ EFTPC (n M) } & \multirow[b]{2}{*}{ Compound Name } & \multirow[b]{2}{*}{ Class } & \multicolumn{3}{|c|}{$\mathrm{pIC}_{50}$} & \multirow[b]{2}{*}{ EFTPC (n M) } \\
\hline & & $h E R G$ & $I_{K s}$ & $I_{C a L}$ & & & & $h E R G$ & $I_{K s}$ & $I_{C a L}$ & \\
\hline Ajmaline & 1 & 5.98 & - & - & 1500 & Me tronidaz ole & 3 & 2.87 & - & 3.75 & 210336 \\
\hline Amiodarone & 1 & 6.38 & 5.59 & 6.57 & 63.5 & Nelfinavir & 3 & 4.90 & 4.10 & - & 60.25 \\
\hline Astemizole & 1 & 8.22 & - & 5.98 & 0.3 & Paroxetine & 3 & 5.72 & - & 5.41 & 12.7 \\
\hline Bepridil & 1 & 7.16 & 5.10 & 6.08 & 33 & Quetiapine & 3 & 5.42 & - & 4.98 & 461.9 \\
\hline Chlorpromazine & 1 & 5.81 & 3.00 & 4.15 & 66.4 & Ranolazine & 3 & 4.90 & 2.72 & 3.51 & 2311 \\
\hline Cilostazol & 1 & 4.86 & - & 4.04 & 162.4 & Solifenacin & 3 & 6.60 & 4.50 & 5.20 & 3.47 \\
\hline Cisapride & 1 & 7.68 & 5.47 & 4.56 & 4.9 & Voriconazole & 3 & 3.31 & - & 3.38 & 5699 \\
\hline Clarithromycin & 1 & 4.06 & - & - & 4011 & Alvimopan & 4 & 3.10 & 4.30 & 5.30 & 5.17 \\
\hline Disopyramide & 1 & 5.00 & 4.09 & 4.96 & 4713 & Ambrisentan & 4 & 3.30 & 3.30 & 2.60 & 75.3 \\
\hline Dofetilide & 1 & 7.75 & - & - & 2 & Ceftriaxone & 4 & 3.35 & - & 3.81 & 13704 \\
\hline Domperidone & 1 & 6.88 & - & - & 5.79 & Darifenacin & 4 & 7.10 & 4.70 & 2.80 & 0.27 \\
\hline Done pezil & 1 & 6.16 & - & 4.47 & 6.56 & Darunavir & 4 & 3.80 & 3.50 & - & 862.5 \\
\hline Dronedarone & 1 & 7.29 & 5.00 & 6.75 & 0.79 & Deferasirox & 4 & 2.40 & - & 3.00 & 1403 \\
\hline Drope ridol & 1 & 7.22 & - & 5.12 & 16 & Desvenlafaxine & 4 & 3.60 & - & - & 3901 \\
\hline Flecainide & 1 & 5.82 & - & 4.57 & 1448 & Diaze pam & 4 & 4.27 & - & 4.52 & 29 \\
\hline Halofantrine & 1 & 6.42 & - & 5.72 & 172 & Diltiazem & 4 & 4.88 & - & 6.12 & 120.6 \\
\hline Haloperidol & 1 & 7.44 & - & 5.77 & 3.6 & Doxorubicin & 4 & 3.00 & 5.32 & - & 4646 \\
\hline Ibutilide & 1 & 7.75 & - & 4.20 & 22 & Duloxetine & 4 & 5.42 & 5.00 & 5.55 & 9.22 \\
\hline Levofloxacin & 1 & 3.37 & - & - & 30932 & Ebastine & 4 & 6.14 & 6.10 & - & 0.14 \\
\hline Me thadone & 1 & 5.46 & - & 4.43 & 507 & Eltrom bopag & 4 & 6.20 & - & - & 158.9 \\
\hline Moxifloxacin & 1 & 4.11 & 3.80 & 3.40 & 6228 & Etravirine & 4 & 3.80 & 2.90 & - & 3.38 \\
\hline Ondanse tron & 1 & 6.09 & 3.00 & - & 178.6 & Everolimus & 4 & 3.30 & 4.00 & - & 8.03 \\
\hline Procainamide & 1 & 3.57 & - & 3.41 & 35058 & Lamivudine & 4 & 2.69 & - & 4.27 & 1446 \\
\hline Quinidine & 1 & 5.90 & 4.36 & 4.81 & 2960 & Lamotrigine & 4 & 3.60 & 3.80 & 2.80 & 19083 \\
\hline Sotalol & 1 & 3.29 & - & - & 18358 & Linezolid & 4 & 2.94 & - & 3.98 & 24856 \\
\hline Sparfloxacin & 1 & 4.66 & - & 4.05 & 1766 & Loratadine & 4 & 5.22 & - & 4.94 & 0.4 \\
\hline Tedisamil & 1 & 6.66 & 5.19 & - & 85 & Maraviroc & 4 & 4.40 & 4.20 & - & 415 \\
\hline Terfenadine & 1 & 7.30 & 5.36 & 6.51 & 9 & Mibe fradil & 4 & 5.77 & 4.93 & 6.29 & 12 \\
\hline Terodiline & 1 & 6.71 & 4.56 & 4.87 & 11.23 & Mitoxantrone & 4 & 3.27 & - & 4.65 & 225 \\
\hline Thioridazine & 1 & 6.70 & 4.99 & 5.88 & 979 & Nebivolol & 4 & 6.50 & 4.80 & - & 1 \\
\hline Clozapine & 2 & 5.64 & - & 5.44 & 70.8 & Nifedipine & 4 & 3.87 & 3.44 & 7.28 & 32.3 \\
\hline Dasatinib & 2 & 4.80 & 3.60 & 3.40 & 20.4 & Nisoldipine & 4 & 4.64 & 4.40 & 7.10 & 0.10 \\
\hline Lapatinib & 2 & 6.00 & 3.60 & 2.70 & 41.8 & Palonosetron & 4 & 5.70 & 4.30 & 3.40 & 1.04 \\
\hline Nilotinib & 2 & 6.90 & 3.40 & 3.70 & 60 & Pentobarbital & 4 & 2.84 & 3.72 & 3.52 & 5171 \\
\hline Ofloxacin & 2 & 2.85 & - & - & 8656 & Phenytoin & 4 & 3.83 & 3.00 & 4.66 & 3964 \\
\hline Paliperidone & 2 & 5.90 & 3.60 & 3.40 & 68.9 & Propranolol & 4 & 5.09 & 3.00 & 4.71 & 10.1 \\
\hline Risperidone & 2 & 6.63 & 5.01 & 4.14 & 6.96 & Raltegravir & 4 & 3.11 & 4.60 & 3.61 & 7000 \\
\hline Saquinavir & 2 & 4.77 & - & 5.72 & 334 & Ribavirin & 4 & 3.02 & - & 3.21 & 15069 \\
\hline Sunitinib & 2 & 6.60 & 4.20 & 4.10 & 19 & Sildenafil & 4 & 4.50 & 3.40 & 4.00 & 100 \\
\hline \multirow[t]{5}{*}{ Tol te rodine } & 2 & 7.90 & 4.10 & - & 0.39 & Silodosin & 4 & 5.10 & 3.60 & 3.10 & 11.2 \\
\hline & & & & & & Sitagliptin & 4 & 3.83 & 3.10 & 3.83 & 589 \\
\hline & & & & & & S-oxybutynin & 4 & 4.92 & 4.54 & 4.79 & 0.05 \\
\hline & & & & & & Tadalafil & 4 & 4.00 & 3.80 & - & 50.1 \\
\hline & & & & & & Telbivudine & 4 & 0.80 & 3.60 & - & 14731 \\
\hline
\end{tabular}

${ }^{a}$ References for all the values in this table are given in the Supporting Information (Table S1).

considered by different international initiatives such as the comprehensive in vitro pro-arrhythmia assay (CiPA). ${ }^{22}$

Recent studies suggest that prediction of arrhythmogenicity could be improved by considering the effects of drugs on multiple ion channels, ${ }^{9,12,13,16}$ the therapeutic plasma drug concentrations, ${ }^{23,24}$ and the use of biophysically detailed mathematical models of cardiac electrical activity. ${ }^{25-29}$

In a previous work, ${ }^{13}$ we introduced a multiscale simulation system to predict drug-induced cardiotoxicity by considering the effects of drugs in two main transmembrane ionic currents: $I_{\mathrm{Kr}}$ and the slow component of the delayed rectifier current $\left(I_{\mathrm{Ks}}\right)$. Here, we create an in silico tool for early detection of drug-induced proarrhythmic risk that provides a new index called $\mathrm{Tx}$, which summarizes in a single parameter the block of different channels in the context of the relevant drug concentration. Action potential duration (APD) and QT prolongations were simulated using the state-of-the-art human ventricular action potential model ( $\mathrm{O}^{\prime} \mathrm{Hara}$ et al. model ${ }^{30}$ ) and taking into account the effect of the block of three transmembrane ionic currents: fast and slow components of the potassium delayed current $\left(I_{\mathrm{Kr}}\right.$ and $\left.I_{\mathrm{Ks}}\right)$ and L-type calcium current $\left(I_{\mathrm{CaL}}\right)$. The simulations have been run over a wide range of concentrations and block values for these currents thus precomputing the cardiac effect that most drug candidates could produce.

The system was used to perform a retrospective cardiac safety study of 84 compounds, 40 of which present torsadogenic properties, according to the information provided by CredibleMeds. ${ }^{31}$ Our results reveal that the Tx index could be a much better predictor of the torsadogenic risk than criteria based solely on the concentration for 50\% hERG inhibition (hERG $\mathrm{IC}_{50}$ ). Therefore, assessment of drug-induced proarrhythmicity of a compound could be improved by using the $\mathrm{Tx}$ index based on in silico APD and QT prolongation methods. Indeed, knowing the $\mathrm{IC}_{50} \mathrm{~s}$ for $I_{\mathrm{Kr}}, I_{\mathrm{Ks}}$, and $I_{\mathrm{CaL}}$ of a compound, our method can be applied to obtain an estimate of the maximum safe plasma free concentration. 

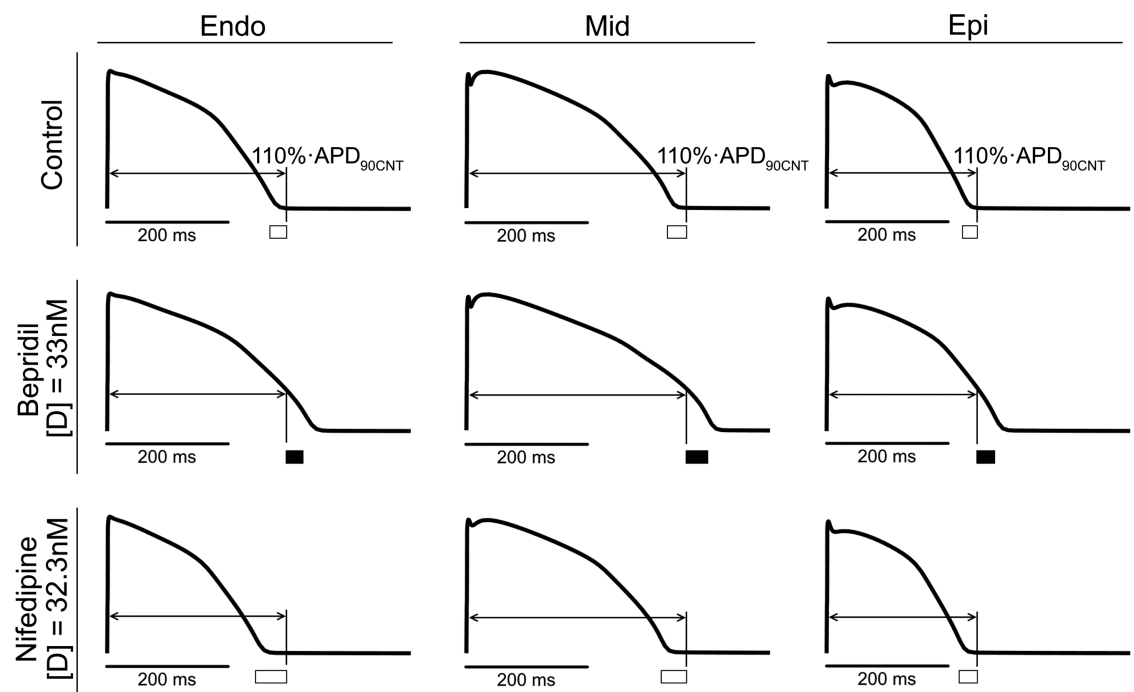

Figure 1. Simulated time course of the action potential of isolated endocardial (Endo), midmyocardial (Mid), and epicardial (Epi) cells (from left to right) under control conditions and in the presence of bepridil $(33 \mathrm{nM})$ and nifedipine (32.3 nM) (from top to bottom). Vertical lines in each panel correspond to $10 \%$ prolongation of the control $\mathrm{APD}_{90}\left(\mathrm{APD}_{90 \mathrm{CNT}}\right)$.

\section{METHODS}

2.1. Cellular and Tissue in Silico Simulations. The action potential (AP) of human ventricular cells was simulated using the human ventricular AP model proposed by O'Hara et al. $\left(\mathrm{ORd}^{30}\right)$. The block of $I_{\mathrm{Kr}}, I_{\mathrm{Ks}}$, and $I_{\mathrm{CaL}}$ provoked by a drug concentration $(D)$ on the ionic current $(i)$ was simulated by including the fraction of unblocked channels $\left(1-b_{i}\right)$ in their formulations and was calculated using the standard sigmoid dose-response curve (eq 1), parametrized using the halfmaximal response dose for each current $\left(\mathrm{IC}_{50, i}\right)$ and considering a Hill coefficient of 1 , as in previous studies: ${ }^{12,13,16,20}$

$$
\frac{I_{i}(D)}{I_{i}}=\frac{1}{1+\frac{D}{\mathrm{IC}_{50, i}}}=1-b_{i}
$$

The effects of drugs on $I_{\mathrm{Kr}}$ (hERG), $I_{\mathrm{Ks}}$ (KCNQ1), and $I_{\mathrm{CaL}}$ (Cav1.2) currents have been analyzed in previous simulation studies using this simple pore block model. ${ }^{11-13,19,32,33}$ APDs were sampled at $90 \%$ repolarization $\left(\mathrm{APD}_{90}\right)$ in isolated epicardial, midmyocardial, and endocardial myocytes at $1 \mathrm{~Hz}$, while pseudo-ECGs were computed using a one-dimensional (1D) tissue model of a transmural wedge preparation. The 1D model was composed by 60 endocardial cells, 45 midmyocardial cells, and 60 epicardial cells ${ }^{30}(100 \mu \mathrm{m}$ long each cell $)$, as defined in the ORd model. Models were paced at $1 \mathrm{~Hz}$, as this frequency is widely used in simulations of the human cardiac electrical activity performed to predict the proarrhythmic risk of drugs. ${ }^{12,16,20,28,34}$ The stimulation train of square transmembrane current pulses of $0.5 \mathrm{~ms}$ duration and 1.5 -fold the diastolic threshold was delivered at the isolated cells and at the endocardial end of the 1D model. The propagation of the AP was described by the following nonlinear reaction diffusion eq (eq 2 ):

$$
C_{\mathrm{m}} \frac{\partial V_{\mathrm{m}}(x, t)}{\partial t}+\sum I_{\text {ion }}+\frac{a}{2} \frac{\partial}{\partial x}\left(\frac{1}{R_{\mathrm{i}}(x)} \frac{\partial V_{\mathrm{m}}(x, t)}{\partial x}\right)=0
$$

where $C_{\mathrm{m}}$ is the membrane capacitance, $a$ stands for the radius of the fiber, $\sum I_{\text {ion }}$ represents the sum of all the ionic currents flowing through the cellular membrane, and $R_{\mathrm{i}}$ is the intracellular resistivity.

Isolated epicardial, midmyocardial, and endocardial cellular models were paced with 1000 pulses at $1 \mathrm{~Hz}$ in order to achieve the steady state in control. The steady state of the $1 \mathrm{D}$ model in control conditions was reached by applying a train of 100 pulses at $1 \mathrm{~Hz}$, the initial conditions for each cell type being the steady state variables obtained from the previously stabilized isolated cellular models. To reach the steady state under drug exposure, the fraction of unblocked channels was introduced in the models, and they were paced for 100 additional pulses (1 $\mathrm{Hz}$ ). For both cellular and 1D models, the results used in our study correspond to the 100th pulse of the last stimulation period.

The QT interval in the pseudo-ECG was computed as the interval between the onset of the QRS complex and the point where the end of the $T$ wave reached the baseline. The pseudoECG was measured at $2 \mathrm{~cm}$ from the epicardial end. Fifteen cells from both ends of the fiber were excluded from the gradient measurement to eliminate boundary effects. ${ }^{30}$

2.2. Drug Data Set. The proarrhythmic risk of 84 compounds (see Table 1) was assessed using our in silico $\mathrm{APD}_{90}$ and QT prolongation assays. For these drugs, clinical human torsadogenic risk classification was taken from CredibleMeds, which is on the current AZCERT's Web site $^{31}$ defining the following categories: class 1 , compounds with risk of TdP; class 2 , compounds with possible risk of TdP; class 3, compounds with conditional risk of TdP; and class 4, drugs that should be avoided by patients with congenital LQTS. We defined the 40 class 1 and 2 compounds as torsadogenic due to their ability to produce $\mathrm{TdP}$ on their own, while the 44 class 3 and 4 compounds were defined as nontorsadogenic as they require specific interactions to produce TdP. According to Mirams et al., ${ }^{12}$ ajmaline was included in class 1. Drugs for which no evidence of $\mathrm{TdP}$ was found in CredibleMeds were also included in class 4 . $\mathrm{IC}_{50}$ values for $I_{\mathrm{Kr}}, I_{\mathrm{Ks}}$, and $I_{\mathrm{CaL}}$ and EFTPC values were obtained from public databases, like DailyMed, PubChem, and DrugBank, when available. Otherwise, the values were taken from the scientific literature. EFTPC values were used either directly from the source or 
calculated from peak plasma concentrations derived from the administration of the drug alone (see Supporting Information, Table S1). We consistently selected the worst-case scenario by searching for the highest dosage regimen proposed by the labels. $\mathrm{IC}_{50}$ values registered in mammalian tissues have been selected when available, avoiding those extracted from Xenopus oocytes. Median values were used when multiple $\mathrm{IC}_{50}$ values were presented. Dashed $\mathrm{IC}_{50}$ boxes mean that no value was found in public databases either in the scientific literature for these compounds. In those cases, our simulations assume no block of the corresponding channel.

\section{RESULTS}

3.1. APD and QT. The effect of the 84 selected drugs on the endocardial, midmyocardial, and epicardial $\mathrm{APD}_{90}$ was simulated using the $\mathrm{pIC}_{50}$ values for hERG, $I_{\mathrm{Ks}}$, and $I_{\mathrm{CaL}}$ and the EFTPC for each compound (see all detailed results in the Supporting Information, Table S2). Figure 1 illustrates some examples of the simulated APs of isolated cells (from left to right) under control conditions and in the presence of bepridil $([\mathrm{D}]=\mathrm{EFTPC}=33 \mathrm{nM})$ and nifedipine $([\mathrm{D}]=\mathrm{EFTPC}=32.3$ $\mathrm{nM}$ ) (from top to bottom). Vertical lines in each panel correspond to $10 \% \mathrm{APD}_{90}$ prolongation. Simulated endocardial, midmyocardial, and epicardial control $\mathrm{APD}_{90} \mathrm{~s}$ are 266.4, 330.3, and $223.8 \mathrm{~ms}$, respectively. This figure shows that the $\mathrm{APD}_{90}$ is markedly prolonged under exposure to the EFTPC of the torsadogenic compound bepridil $\left(\mathrm{APD}_{90} \mathrm{~s}\right.$ are 331.1, 398.0, and $278.0 \mathrm{~ms}$, and $\mathrm{APD}_{90}$ increments are $24.3 \%, 20.5 \%$, and $24.2 \%$, respectively). On the contrary, $\mathrm{APD}_{90}$ is reduced under exposure to the EFTPC of the nontorsadogenic compound nifedipine (endocardial, midmyocardial, and epicardial $\mathrm{APD}_{90} \mathrm{~s}$ are 236,6, 315.7, and $216.1 \mathrm{~ms}$, and $\mathrm{APD}_{90}$ increments are $-11.2 \%,-4.4 \%$, and $-3.4 \%$, respectively).

As the QT interval prolongation is used in the assessment of drug proarrhythmic risks, the effects of the 84 compounds on the QT interval was also simulated (see Supporting Information, Table S2). Figure 2 depicts the virtual onedimensional transmural tissue (top panel) and the simulated pseudo-ECG at $2 \mathrm{~cm}$ from the epicardial end, for control, and for bepridil and nifedipine at their respective EFTPC (second to bottom panel). Simulated control $\mathrm{QT}$ interval $\left(\mathrm{QT}_{\mathrm{CNT}}\right)$ is $313 \mathrm{~ms}$. Vertical lines in each panel correspond to $10 \%$ prolongation of the $\mathrm{QT}_{\mathrm{CNT}}{ }^{13}$ The $\mathrm{QT}$ interval is markedly prolonged under exposure to the EFTPC of bepridil (QT interval $=376 \mathrm{~ms}$ and QT interval increment $=20.1 \%$ ), while it is reduced under exposure to the EFTPC of nifedipine (QT interval $=299 \mathrm{~ms}$ and $\mathrm{QT}$ interval increment $=-4.5 \%)$. The effects of these two drugs on the QT interval are in agreement with those obtained in the $\mathrm{APD}_{90} \mathrm{~S}$ of isolated cells (Figure 1).

To facilitate the prediction of the potential proarrhythmic risk of a wide range of drugs without carrying out a timeconsuming simulation, the effect on the simulated action potential durations of the three types of myocardial cells and of QT intervals were precomputed for a large number of $I_{\mathrm{Kr}}, I_{\mathrm{Ks}}$, and $I_{\mathrm{CaL}}$ block values and concentration combinations. The 206.766 simulations of the electrical activity of isolated myocytes were run at a pacing rate of $1 \mathrm{~Hz}$, varying the ratios of drug concentration over the $\mathrm{IC}_{50}$ of each considered ion channel $\left(I_{\mathrm{Kr}}, I_{\mathrm{Ks}}\right.$, and $\left.I_{\mathrm{CaL}}\right)$, ranging from $10^{-3}$ to $10^{1}$ with a $10^{0.1}$ step increment. 7072 simulations were performed to obtain the QT intervals at $1 \mathrm{~Hz}$. In this case, the ratios of drug concentration over the $\mathrm{IC}_{50}$ ranged from $10^{-2}$ to $10^{1}$ for $I_{\mathrm{Kr}}$ and $I_{\mathrm{Ks}}$ and from $10^{-2}$ to $10^{0}$ for $I_{\mathrm{CaL}}$. Additionally, step
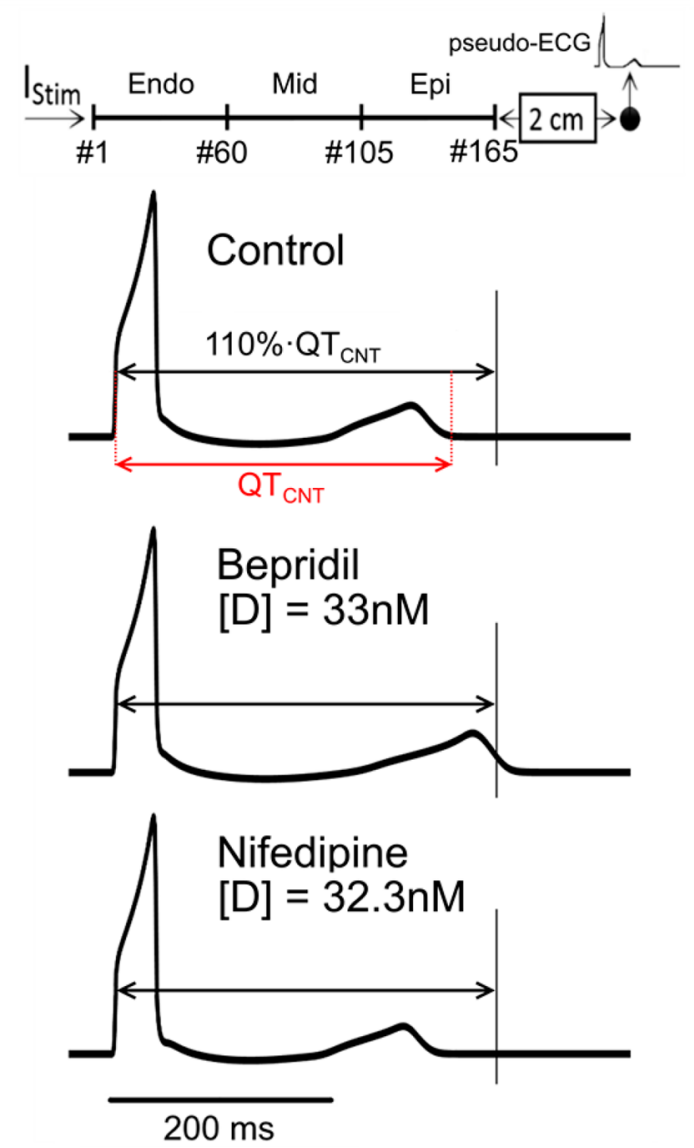

Figure 2. Representation of the virtual transmural 1D tissue (top panel) and the time course of the pseudo-ECG for control and for bepridil and nifedipine at their therapeutic concentrations (second to bottom panel), respectively. Black vertical lines in each panel correspond to $10 \%$ prolongation of the control QT interval $\left(\mathrm{QT}_{\mathrm{CNT}}\right)$, whose measurement is shown in red.

increments depended on the ion channel. Specifically, $I_{\mathrm{Kr}}$ step increment was set to $10^{0.2}$ except for the ratios between $10^{-1.2}$ and $10^{-0.2}$, where it was set to $10^{0.1}$, and $I_{\mathrm{Ks}}$ and $I_{\mathrm{CaL}}$ step increments were set to $10^{0.25}$ and $10^{0.2}$, respectively.

Figure 3 shows a 3D representation of the surface (blue striped) corresponding to the $110 \%$ of control $\mathrm{APD}_{90}(110 \%$ $\mathrm{CNT}$ ) for endocardial (top left), midmyocardial (top right), and epicardial (bottom left) cells as well as the QT interval (bottom right). Coordinate axes represent the logarithm of the ratio of the drug concentration (D) over the $\mathrm{IC}_{50}$ of each channel, which expresses how much larger the concentration used in the simulation is than the concentration known to block $50 \%$ of channels. $I_{\mathrm{Kr}}$ and $I_{\mathrm{Ks}}$ block increases $\mathrm{APD}_{90} \mathrm{~s}$ and the QT interval, while $I_{\mathrm{CaL}}$ block decreases them, so the back bottom left zones of the blue surface correspond to $\mathrm{APD}_{90} \mathrm{~s}$ and $\mathrm{QT}$ intervals longer than $110 \%$ of control values while front top right zones correspond to $\mathrm{APD}_{90} \mathrm{~s}$ and $\mathrm{QT}$ intervals shorter than $110 \%$ of control values. Those compounds that fall left to the $110 \%$ blue surfaces prolong the $\mathrm{APD}_{90}$ or the QT interval more than $10 \%$, like disopyramide (red triangle) and bepridil (red cube) at EFTPC. Conversely, those compounds that fall right of the $110 \%$ blue surfaces prolong the $\mathrm{APD}_{90}$ or QT less than $10 \%$, like nontorsadogenic drugs raltegravir (green circle) and phenytoin (green cube), at EFTPC. 

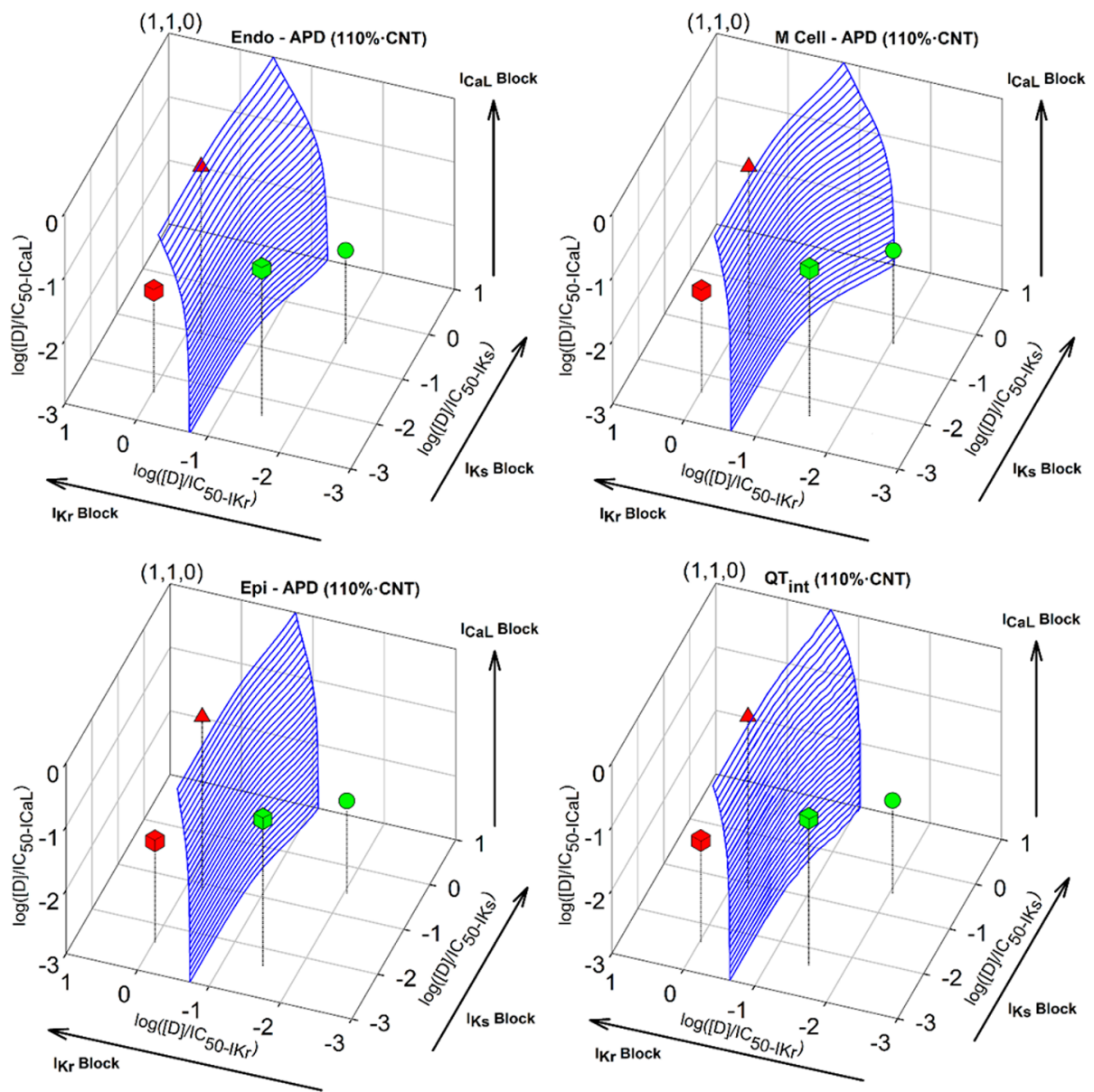

Figure 3. 3D representation of the surface (blue striped) corresponding to the $110 \%$ of the control endocardial (top left), midmyocardial (top right), and epicardial (bottom left) $\mathrm{APD}_{90}$ and QT interval (bottom right) together with selected torsadogenic drugs, namely disopyramide (red triangle), bepridil (red cube), and nontorsadogenic drugs, namely raltegravir (green circle) and phenytoin (green cube). Coordinate axes represent the logarithm of the ratio of the drug concentration over the $\mathrm{IC}_{50}$ of each channel $\left(\log \left([\mathrm{D}] / \mathrm{IC}_{50}\right)\right)$.

It is possible to observe how the surfaces representing the $110 \%$ of control of the endocardial and epicardial $\mathrm{APD}_{90}$ and of the QT interval are quite similar, while the midmyocardial $\mathrm{APD}_{90}$ is more sensitive to $I_{\mathrm{Ks}}$ block. Also, within the computed limits, $I_{\mathrm{Kr}}$ block exerts the strongest influence on $\mathrm{APD}_{90}$ and QT prolongation among the three channels. Indeed, approximately $9 \%$ of $I_{\mathrm{Kr}}$ channels $\left(\log \left([\mathrm{D}] / \mathrm{IC}_{50-\mathrm{IKr}}\right)=-1\right)$ should be blocked in order to reach the $110 \%$ of the endocardial $\mathrm{APD}_{90}$ and the QT interval. However, when $I_{\mathrm{CaL}}$ is inhibited, the percentage of blocked $I_{\mathrm{Kr}}$ channels needed to prolong the $10 \%$ of the $\mathrm{APD}_{90}$ and the $\mathrm{QT}$ interval increases. For example, for $50 \%$ of $I_{\mathrm{CaL}}$ inhibition $\left(\log \left([\mathrm{D}] / \mathrm{IC}_{50-\mathrm{ICaL}}=0\right)\right)$, the percentage of $I_{\mathrm{Kr}}$ block reaches $24 \%$, with no block of $I_{\mathrm{Ks}}\left(\log \left([\mathrm{D}] / \mathrm{IC}_{50-\mathrm{IKs}}\right.\right.$ $=-3)$ ).

3.3. New Indices for TdP Risk Assessment. Figure 4 represents the relationship between the $\mathrm{pIC}_{50} \mathrm{hERG}$ values $\left(-\log \mathrm{IC}_{50} \mathrm{hERG}\right)$ and the risk classification for the 84 drugs, 40 torsadogenic and 44 nontorsadogenic drugs (positive response $\mathrm{pIC}_{50}>6$ ). This figure shows that 39 out of the 44 nontorsadogenic compounds (class 4 (dark-green) and class 3 (bright-green) compounds) have $\mathrm{pIC}_{50} \mathrm{hERG}$ values smaller than 6 (vertical black line), and their nontorsadogenic risk would be well predicted by this criterion, while 5 out of those 44 would be misclassified as positives. Conversely, 18 out of the 40 torsadogenic drugs (class 1 (red) and class 2 (orange)) have $\mathrm{pIC}_{50}$ hERG values smaller than 6 , resulting in false negatives.

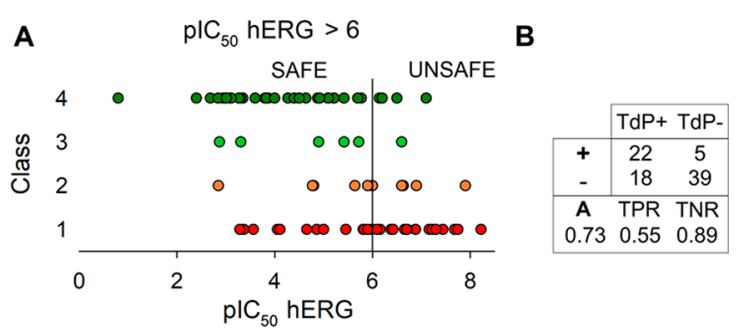

Figure 4. (A) Relationship between the $\mathrm{pIC}_{50} \mathrm{hERG}$ values $\left(-\log \mathrm{IC}_{50}\right.$ hERG) and the color-coded risk classification for the 84 drugs used in this study: class 1 (red) and class 2 (orange), class 3 (bright-green), and class 4 (dark-green). The vertical line corresponds to $\mathrm{pIC}_{50} \mathrm{hERG}$ = 6. (B) confusion matrix of the $\mathrm{pIC}_{50} \mathrm{hERG}$ criterion.

The performance of this test is described by the confusion matrix included in Figure 4B. The performance for the decision $\mathrm{pIC}_{50}$ hERG > 6 was: sensitivity (true positive rate) of $55 \%$, specificity (true negative rate) of $89 \%$, and accuracy (true rate) of $73 \%$.

To summarize the block of the different channels in the range of the relevant drug concentration in a single parameter, we define the new index, $\mathrm{Tx}$, as the ratio of drug concentrations leading to a $10 \%$ prolongation of the $\mathrm{APD}_{90}$ or $\mathrm{QT}\left(\left[D_{10}\right]\right)$ over the maximum effective free therapeutic plasma concen- 
tration (EFTPC), which is the peak concentration of free (unbound) drug in blood plasma at maximum therapeutic dose:

$$
\mathrm{Tx}=\frac{\left[\mathrm{D}_{10}\right]}{\text { EFTPC }}
$$

This index was quantified using the following method. The $\mathrm{APD}_{90}$ and $\mathrm{QT}$ at maximum effective free therapeutic concentration $(D=$ EFTPC) of a certain compound were looked up in the precomputed matrices (see Figure 3). Then, the value of $\log \left([\mathrm{D}] / \mathrm{IC}_{50}\right)$ for each channel was increased or reduced by 0.1 if the $A P D_{90}$ or $\mathrm{QT}$ was shorter or longer than $110 \%$ of control value, respectively, and the $\mathrm{APD}_{90}$ or QT was evaluated at this new concentration in the matrices. These two steps were repeated until the $110 \%$ of $\mathrm{APD}_{90}$ or $\mathrm{QT}$ was reached, denoting the latter concentration as $\left[\mathrm{D}_{10}\right]$. Finally, $\mathrm{Tx}$ was computed as the ratio of $\left[D_{10}\right]$ over the maximum therapeutic concentration $\left(\mathrm{Tx}=\left[\mathrm{D}_{10}\right] /\right.$ EFTPC). Because of the resolution of the QT matrix, if a specific value was not computed, the closest available value was used instead.

The previously explained method was used to assign a $\mathrm{Tx}$ value to every drug in Table 1 (see Supporting Information, Table S2). To select the Tx-APD values that best classify drugs for torsadogenic risk, receiver operating characteristic (ROC) curves were constructed. Figure 5 shows the ROC curves for

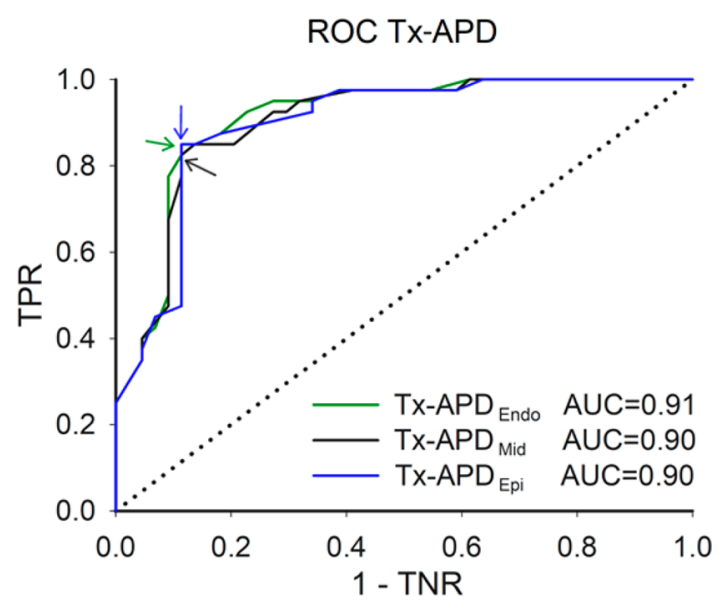

Figure 5. In silico isolated cellular Tx-APD assays. ROC curves for the Tx-APD and optimal cutoff points where sensitivity and specificity are maximal (arrows) for the Tx-APD $\mathrm{Ando}_{\text {Endo }}$ (green), Tx-APD ${ }_{\text {Mid }}$ (black), and Tx-APD $\mathrm{Epi}_{\text {(blue }}(8,8$, and 6.4 , respectively). The area under the curves (AUC) is also indicated.

endocardial Tx-APD $\mathrm{D}_{\text {Endo }}$ (green), midmyocardial $\mathrm{Tx}-\mathrm{APD}_{\mathrm{Mid}}$

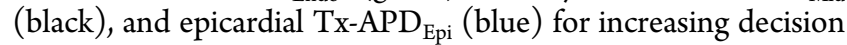
thresholds and the optimal cutoff points where sensitivity and specificity are maximal (arrows). This figure illustrates that the ROC curves are very similar, being the area under these curves (AUC) of $0.91,0.90$, and 0.90 for the Tx-APD $D_{\text {Endo }}$ Tx-APD ${ }_{\text {Mid }}$ and $\mathrm{Tx}-\mathrm{APD}_{\mathrm{Ep}}$, respectively, which are indicative of overall concordance. These indicators would therefore provide similar predictions. The optimal cutoff points of the Tx-APD $\mathrm{Endo}_{\mathrm{Tx}} \mathrm{T}-$ $\mathrm{APD}_{\mathrm{Mid}}$, and Tx-APD $\mathrm{Epi}_{\text {ine }}$, 8 , and 6.4 , respectively. The ROC curves for the Tx-APD $D_{\text {Endo }}$ were also generated at 2 and $0.5 \mathrm{~Hz}$. As they are very similar to the Tx-APD $\mathrm{Endo}_{\text {ROC curve }}$ at $1 \mathrm{~Hz}$ (see Figure S3 in the Supporting Information), we maintained the frequency at $1 \mathrm{~Hz}$.

Figure 6A represents the obtained Tx-APD (see also Supporting Information, Table S2), each row containing compounds belonging to only one CredibleMeds class. Vertical lines were drawn matching the optimal cutoff values whose confusion matrices are summarized in Figure 6B. All these tests led to very similar accuracies $(86-87 \%)$. However, Tx-APD $D_{\text {Endo }}$ and $\mathrm{Tx}-\mathrm{APD}_{\mathrm{Epi}}$ led to $85 \%$ sensitivity and $89 \%$ specificity, while Tx-APD ${ }_{\text {Mid }}$ led to $85 \%$ sensitivity and $86 \%$ specificity.

Similarly, a ROC curve was constructed to study if the index Tx-QT could improve the prediction of torsadogenic risk (Figure 7A). Figure 7B shows the relationship between the TxQT and the risk classification for the 84 drugs (see also Supporting Information, Table S2), which resembles the relationships between the three Tx-APDs and the risk classification for the drugs. The ROC curve for the Tx-QT shows an area under the curve of 0.90 , which is also very similar to the Tx-APDs (Figure 5). The arrow represents the Tx-QT (9.2) that provided optimal overall prediction of the torsadogenic risk, with $85 \%$ sensitivity, $89 \%$ specificity, and $87 \%$ accuracy (see confusion matrix in Figure 7C).

Figure 8 shows the torsadogenic risk classification of the 84 compounds using three different criteria: $\mathrm{pIC}_{50} \mathrm{hERG}>6$ (top panel), Tx-APD Endo $<8.0$ (middle panel) and Tx-QT $<9.2$ (bottom panel). It is worth noting that both $\mathrm{Tx}$ indices misclassified the same 11 compounds from the 84 included in the data set, whereas hERG test misclassified 23 compounds. $\mathrm{Tx}$ indices misclassified as nontorsadogenic (false negative) three class 1 compounds (compounds with risk of TdP), namely cilostazol, donepezil, and dronedarone, and three class 2 compounds (compounds with possible risk of TdP), namely dasatinib, ofloxacin, and saquinavir. Additionally, $\mathrm{Tx}$ indices also misclassified as torsadogenic (false positive) three class 3 compounds (compounds with conditional risk of $\mathrm{TdP}$ ), namely metronidazole, quetiapine, and ranolazine, and two class 4 compounds, namely eltrombopag and lamotrigine.

The predictive power of the Tx indices was assessed by using a leave-one-out cross-validation procedure. We built 84 different data sets (training sets) by removing one drug from

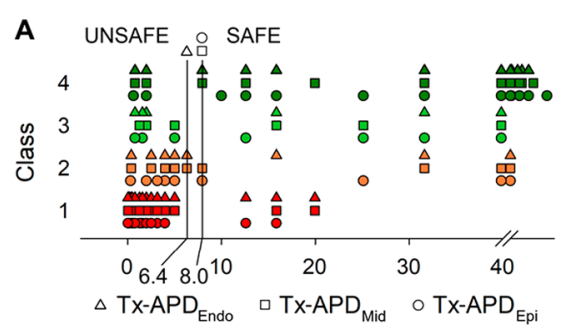

B

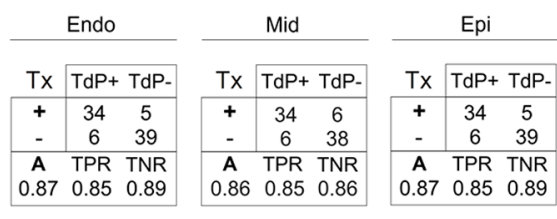

Figure 6. In silico isolated cellular Tx-APD assays. (A) Relationship between the Tx-APD $\mathrm{Endo}_{\mathrm{x}} \mathrm{Tx}-\mathrm{APD}_{\mathrm{Mid}}$, and $\mathrm{Tx}$-APD $\mathrm{Epi}_{\mathrm{Ei}}($ triangles, squares, and circles, respectively) values and the risk classification for the drugs: class 1 (red), class 2 (orange), class 3 (bright-green) and class 4 (dark-green). (B) Confusion matrices for the Tx-APDs. The values of the true positive rate (TPR), true negative rate (TNR), and accuracy (A) are also indicated. 

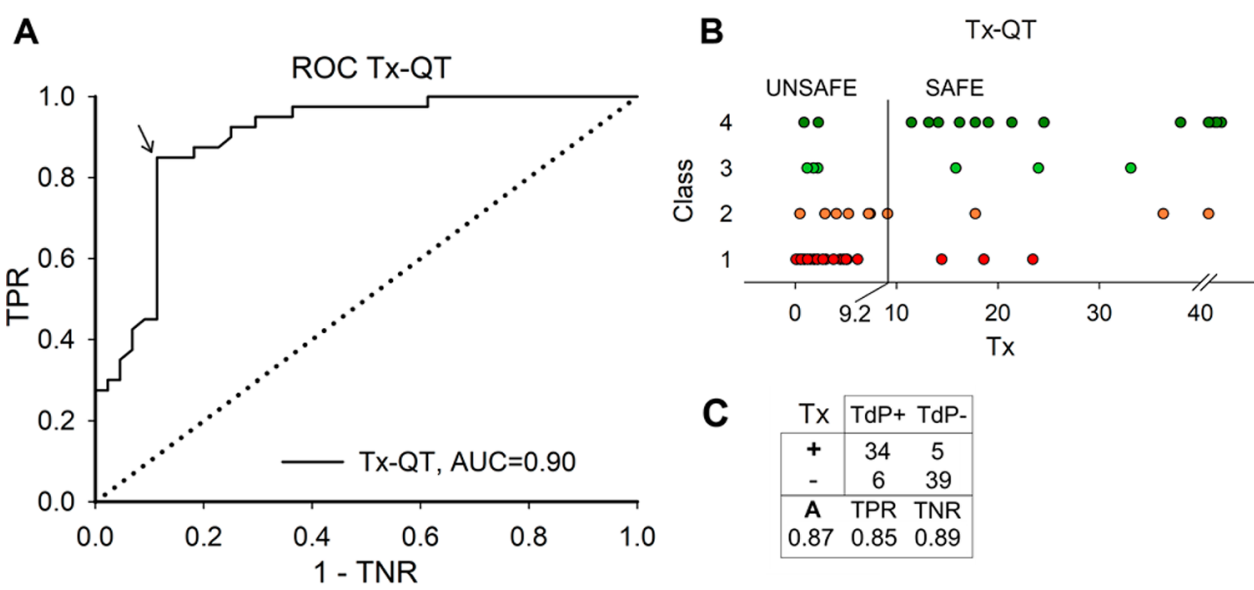

\begin{tabular}{cc|cc|} 
& \multicolumn{1}{c}{ Tx } & TdP + TdP- \\
\hline $\mathbf{t}$ & 34 & 5 \\
- & 6 & 39 \\
\hline A & TPR & TNR \\
0.87 & 0.85 & 0.89 \\
\hline
\end{tabular}

Figure 7. In silico QT interval Tx assays. (A) ROC curves for the Tx-QT and optimal cutoff points where sensitivity and specificity are maximal for the Tx-QT (arrow, 9.2). The area under the curve is also indicated. (B) Relationship between Tx-QT and the risk classification for the drugs: class 1 (red), class 2 (orange), class 3 (bright-green), and class 4 (dark-green). (C) Confusion matrix for the Tx-QT. The values of the true positive rates (TPR), true negative rates (TNR), and accuracies (A) are also indicated.
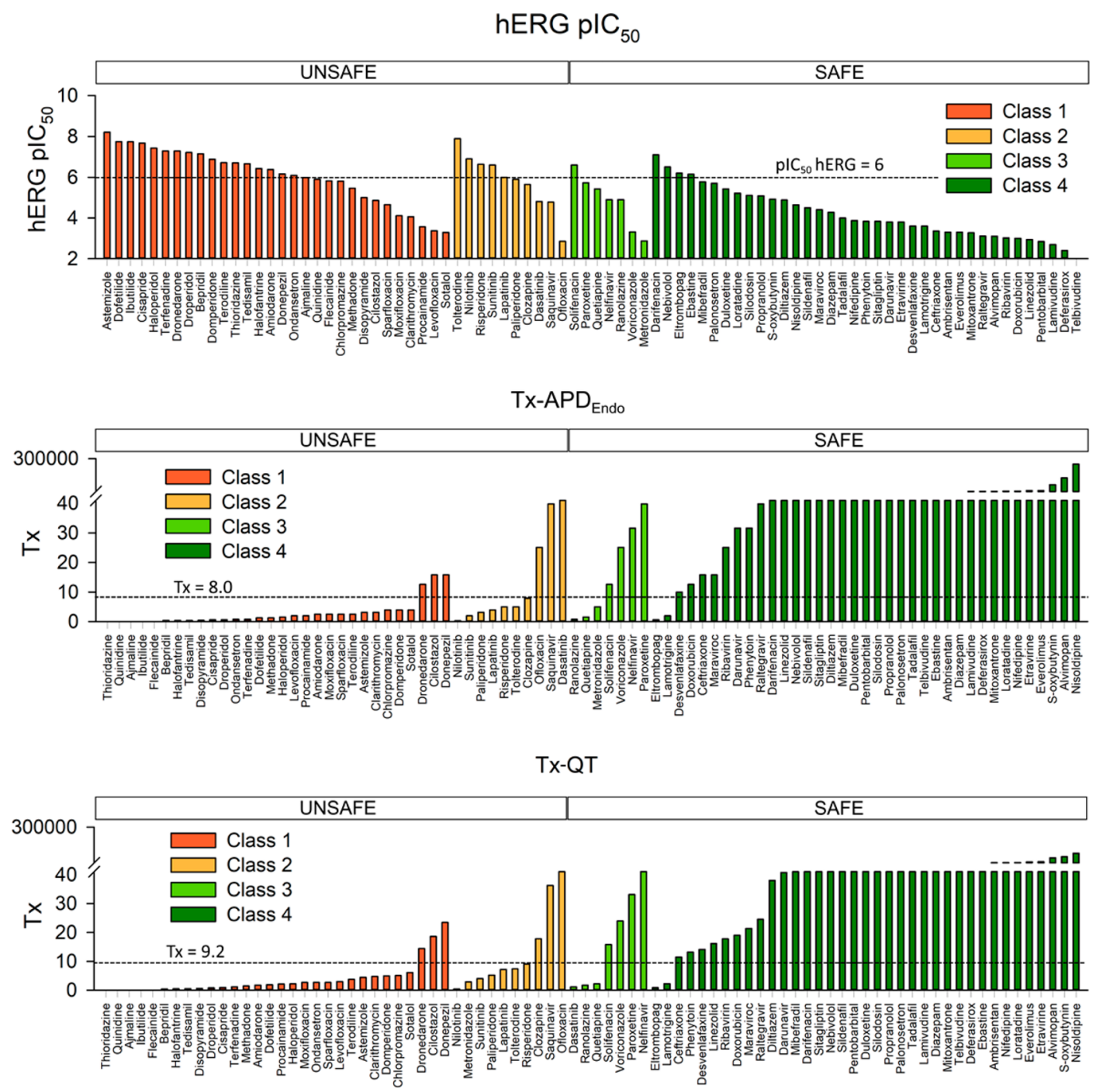

Figure 8. Torsadogenic risk classification of the 84 compounds using $\mathrm{pIC}_{50} \mathrm{hERG}>6$ (top panel), Tx-Endo $<8.0$ (middle panel), and Tx-QT $<9.2$ (bottom panel).

the total compounds each time. ROC curves for the 84 training sets were used to estimate the optimal cutoff $\mathrm{Tx}$ of each training sets (not shown). ROC curves for APD of endocardial, midmyocardial, and epicardial cells and for QT interval present 

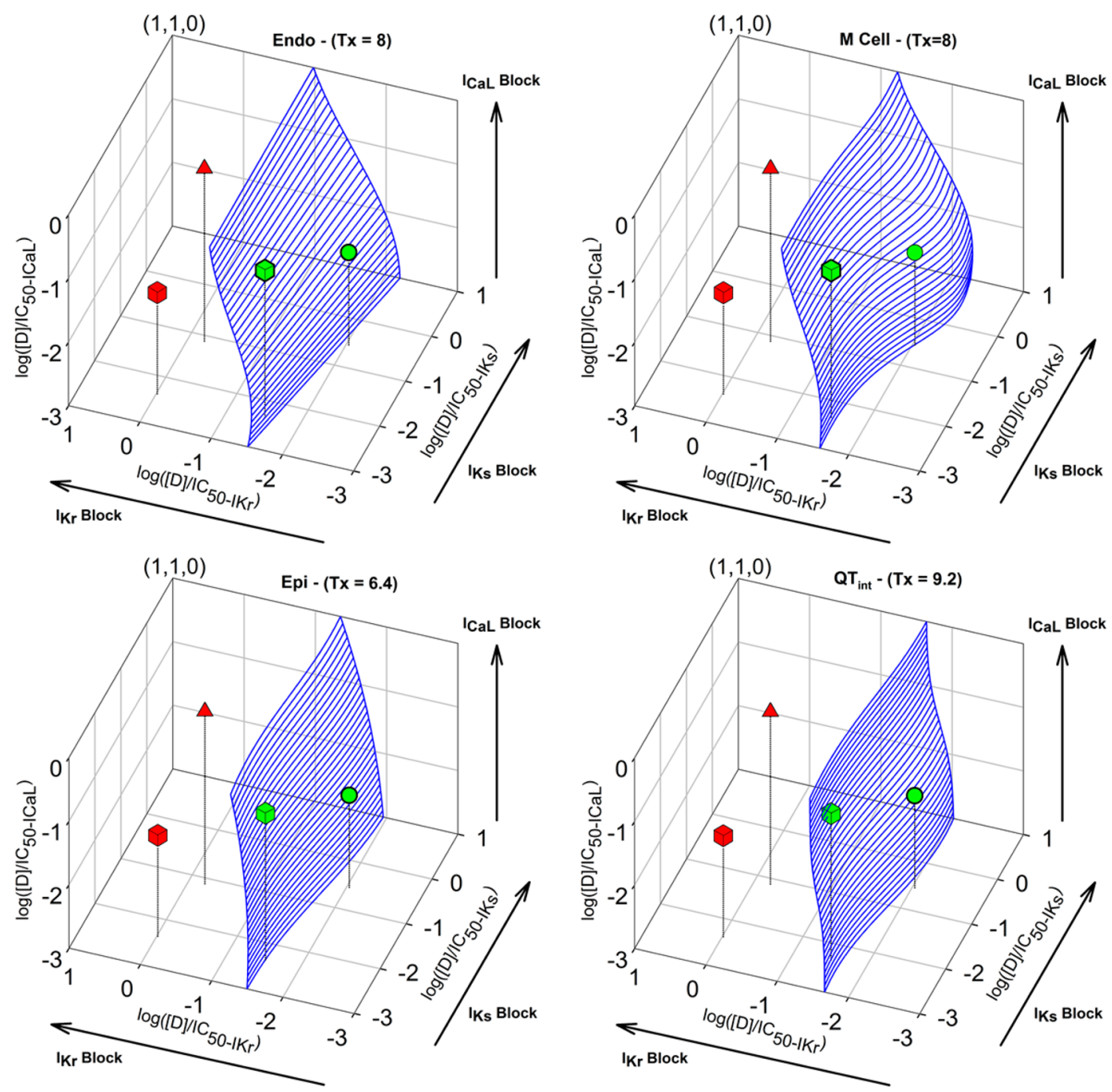

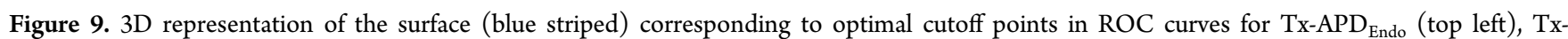
$A P D_{M i d}$ (top right), and $T x-A P D_{\mathrm{Epi}}$ (bottom left) $\mathrm{APD}_{90}$ and $\mathrm{Tx}-\mathrm{QT}$ (bottom right) together with Tx calculated for selected torsadogenic drugs, namely disopyramide (red triangle) and bepridil (red cube), and nontorsadogenic drugs, namely raltegravir (green circle) and phenytoin (green cube). Coordinate axes represent the logarithm of the ratio of the drug concentration over the $\mathrm{IC}_{50}$ of each channel $\left(\log \left([\mathrm{D}] / \mathrm{IC} \mathrm{I}_{50}\right)\right.$ ).

very consistent values, with a cutoff Tx of $7.91 \pm 0.32$ (mean \pm SD), $6.33 \pm 0.18,6.31 \pm 0.00$, and $9.10 \pm 0.19$, a sensitivity of $0.85 \pm 0.01,0.83 \pm 0.01,0.85 \pm 0.01$, and $0.85 \pm 0.01$, a specificity of $0.89 \pm 0.01,0.89 \pm 0.01,0.89 \pm 0.01$, and $0.89 \pm$ 0.01 , and an area under the curve (AUC) of 0.91, 0.90, 0.90, and 0.90 , respectively. For each training set, the "left-out" drug was classified according to its $\mathrm{Tx}$ value. At the end of the procedure, we observed that there was a group of drugs that were misclassified for any of the Tx used in each test. Tx$\mathrm{APD}_{\text {Endo, }} \mathrm{Tx}_{\mathrm{APD}} \mathrm{APid}_{\mathrm{M}}$, and Tx-QT, also misclassified clozapine, as it is the frontier compound when selecting the optimal cutoff Tx in every ROC curve. In summary, approximately $14 \%$ of the drugs are misclassified when any of the Tx indices are used to predict the torsadogenic risk.

Figure 9 shows a 3D representation of the surface (blue striped) corresponding to optimal cutoff points in ROC curves

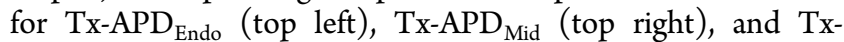
$\mathrm{APD}_{\mathrm{Epi}}$ (bottom left) $\mathrm{APD}_{90}$ and $\mathrm{Tx}-\mathrm{QT}$ (bottom right) together with Tx calculated for selected drugs. Tx matrices were built using the same APD and QT matrices used to generate Figure 3. Tx matrices were constructed to provide a ready-touse tool for Tx assessment for drugs whose $\mathrm{IC}_{50} \mathrm{~s}$ for $I_{\mathrm{Kr}}, I_{\mathrm{Ks}}$, and $I_{\mathrm{CaL}}$, as well as their target concentration, have been characterized.

Matrices corresponding to Figures 3 and 9 are available from the institutional and public repository of the Polytechnic University of Valencia (RIUNET, http://hdl.handle.net/ 10251/90297). Moreover, these matrices were incorporated in a set of predictive models developed within the framework of the eTOX project ${ }^{48}$ and included in the eTOXsys integrated predictive system. ${ }^{49}$ The open access version of these models is made accessible online (http://etoxlab-lqt.upf.edu), allowing reproduction of the results described here as well as to carry out the assessment of the proarrhythmic risk for other substances. In the case of new compounds, as the EFTPC is unknown, Tx matrices can be used to find an estimate of the maximum safe concentration. For this purpose, an initial concentration must be provided instead of the EFTPC, together with the $\mathrm{IC}_{50} \mathrm{~s}$. The Tx matrix for this set of inputs will provide a certain $T x$ value. This Tx value must be compared to the optimal cutoff value of the model. If it is higher, then, the concentration should be increased while if it is lower, it should be decreased. Thus, to find a maximum safe concentration, the process must 
be repeated for several concentrations until the resulting $\mathrm{Tx}$ is equal to the optimal cutoff value of the model.

\section{DISCUSSION}

4.1. Main Findings. In this paper, we describe a new tool for the preclinical assessment torsadogenic risk that provides better predictions that the current state-of-the-art methodology based only on hERG block. The torsadogenic risk assessment is based on a new index, $\mathrm{Tx}$, which integrates the influence of relevant factors and is defined as the ratio of the drug concentration, producing $10 \%$ prolongation of the cellular endocardial, midmyocardial, and epicardial APDs or the QT interval over the maximum EFTPC. This tool employs a large number of values of prolongations of the $\mathrm{APD}_{90}$ and $\mathrm{QT}$ interval caused by the multichannel block of three important ionic membrane currents $\left(I_{\mathrm{Kr}}, I_{\mathrm{Ks}}\right.$, and $\left.I_{\mathrm{CaL}}\right)$, which are predicted by computer simulations of the electrophysiological activity of the cardiac cells. By including these multichannel effects and the maximum EFTPC, misclassifications in a group of 84 compounds using the hERG block criterion were drastically reduced (more than 50\%), from 23 to 11 . In our opinion, adoption of these tools into cardiovascular safety assessment could speed up and reduce the cost of cardiac safety screening in the early stages of drug discovery.

4.2. In Silico $A P D_{90}$ and QT Prolongation Assay versus the hERG Block Test. Standard assessment methodologies focused on hERG block exhibit a poor performance. ${ }^{2,35}$ Indeed, when applying the hERG test (positive response: hERG pIC $_{50}>6$ ) to the 84 compounds considered in this work, it exhibited a sensitivity of $55 \%$, a specificity of $89 \%$, and an accuracy of $73 \%$, which is in close agreement with previous studies. ${ }^{9,36}$ In comparison, the in silico Tx tests described in this study presented an accuracy of $86 \%-87 \%$.

In a previous paper, we showed that the prediction of the TdP risk obtained with the classic hERG block could be improved by simulating the block of $I_{\mathrm{Kr}}$ and $I_{\mathrm{Ks}}$ using a guinea pig AP model. ${ }^{13}$ In the same year, Mirams and co-workers simulated the block of $I_{\mathrm{Kr}}, I_{\mathrm{CaL}}$, and $I_{\mathrm{Na}}$ by 31 drugs with different risks of $\mathrm{TdP}$ and observed that simulation of the human ventricular APD at therapeutic concentrations improved the prediction of Redfern and co-workers. ${ }^{12}$ Both works were pioneers in using computer models to introduce the multichannel effects of drugs for predicting torsadogenic risks. In a previous work, Mirams et al. ${ }^{16}$ also assessed the performance of the prediction of the thorough QT assay in 34 drugs by using several ventricular human models, and they showed that the O'Hara et al. model ${ }^{30}$ exhibited the best performance $(88 \%$ accuracy, $71 \%$ sensitivity, and $100 \%$ specificity when considering a wider range of concentrations). More recently, Lancaster and Sobie ${ }^{20}$ have also evaluated arrhythmia risk using different ventricular human models and have obtained better scores with the O'Hara et al. model ( $89.5 \%$ accuracy, $95.9 \%$ sensitivity, and $81.1 \%$ specificity). In that work, they combined multichannel block simulations of 68 drugs (with a total of 86 cases, as 18 of these drugs were simulated using two different models, each of them constructed with the data of only one of the two sources of experimental data used in that paper) with statistical analysis and machine-learning. They observed that arrhythmia risk is influenced by both the action potential and the intracellular calcium concentrations waveforms.

The better performance of our in silico tool compared to the hERG test cannot be attributed solely to the consideration of the multichannel block but also to the use of compound
EFTPC. Indeed, previous works have shown that torsadogenic risk predictions can be improved by including the estimated free plasma concentration at therapeutic doses of the compound and multichannel block effects. In 2003, Redfern and co-workers observed that a safety factor of 30 of the ratio of the hERG $\mathrm{IC}_{50}$ over the maximum effective free therapeutic plasma concentration could provide good torsadogenic risk prediction for 52 drugs $^{23}$ (77.4\% accuracy, $87.5 \%$ sensitivity, and $68 \%$ specificity). Later, Gintant ${ }^{24}$ proposed the value 45 for the safety margin, defined as the ratio of the hERG $\mathrm{IC}_{50}$ over the mean maximal plasma drug concentrations during thorough QT) studies. Our work extends their findings with a larger data set and the inclusion of the multichannel effects of the compounds.

All in all, our results show that Tx index is a better predictor than the hERG pIC $_{50}$ test (accuracy $\approx 88 \%$ compared with $73 \%$, respectively) and that the results of predictions obtained with the four Tx tests $\left(\mathrm{Tx}-\mathrm{APD}_{\mathrm{Endo}}, \mathrm{Tx}-\mathrm{APD}_{\mathrm{Mid}}\right.$, and Tx-APD $\mathrm{Epi}$ and $\mathrm{Tx}-\mathrm{QT}$ ) are very similar. Moreover, the accuracy of our classification method (87\%) is in line with the scores of the predictions of recent studies, such as Mirams et al. 2014 (88\%), Lancaster and Sobie 2016 (89.5\%), or Kramer et al. (90.9\%), but our study considers a larger number of compounds (84 compared to 34,68 , and 55 , respectively).

Our simulations have shown that $I_{\mathrm{Ks}}$ exerts a small influence on $\mathrm{APD}_{90}$ of isolated cells and $\mathrm{QT}$ prolongation under regular conditions. This is in accordance with the small amplitude of the $I_{\mathrm{Ks}}$ on isolated myocytes from human left ventricles ${ }^{37}$ and with other simulation results. ${ }^{16,30}$ Moreover, our results show that the role of $I_{\mathrm{CaL}}$ block on $\mathrm{APD}_{90}$ of isolated cells and on QT prolongation is relevant although to a lesser extent than $I_{\mathrm{Kr}}$ block. These facts suggest that the experimental and in silico study of the effects of drugs on $I_{\mathrm{Kr}}$ and $I_{\mathrm{CaL}}$ on myocytes isolated from human ventricles is more relevant than drug effects on $I_{\mathrm{Ks}}$. This result is in agreement with the work of Kramer and collaborators who observed that the TdP risk prediction provided by the comparison of the blocking potencies between $I_{\mathrm{Kr}}$ and $I_{\mathrm{CaL}}$ was drastically better than the prediction obtained by the hERG assay in 55 drugs $^{9}$ (90.9\% accuracy, $96.9 \%$ sensitivity, and $82.6 \%$ specificity).

Other works have simulated $\mathrm{APD}_{90}$ changes in isolated endocardial cells of different species such as guinea pigs, ${ }^{13}$ rabbits, dogs, and humans ${ }^{12,16}$ and QT interval changes in rabbit ${ }^{15}$ to predict cardiac safety of torsadogenic drugs. Beattie et al. showed that in silico simulation of the QT prolongation of the rabbit left ventricular wedge assay had a good predictive ability ( $78 \%$ accuracy) of the experimental results of the rabbit left ventricular wedge assay. ${ }^{15}$ We selected the most recent human ventricular AP model for our simulations instead of models of other species with the aim of obtaining more accurate predictions of drug proarrhythmicity risk in humans. ${ }^{38}$ Indeed, comparison between human and other species has revealed a lack of a transient outward current $\left(I_{\text {to }}\right)$ in guinea pig, a small slow delayed rectifier current $\left(I_{\mathrm{Ks}}\right)$ and TdP predisposition in rabbit, and a large $I_{\mathrm{Ks}}$ in guinea pig. ${ }^{39}$ Other studies have shown that human $I_{\mathrm{Ks}}$ differs from that reported in guinea pig, and it better resembles the ventricular canine or rabbit $I_{\mathrm{Ks}}{ }^{37}$

Our work feeds from the progress achieved by the aforementioned works, such as the consideration of the therapeutic concentrations and the multichannel effects, and the use of the most recent human ventricular action potential model and goes a step further by providing a tool for prediction 
of the torsadogenic risk that can be used by the pharmaceutical companies at the early stages of drug development. This tool can be applied when the $\mathrm{IC}_{50}$ s for $I_{\mathrm{Kr}}, I_{\mathrm{Ks}}$, and $I_{\mathrm{CaL}}$ of a new compound are estimated without the need of high performance hardware or cardiac modeling specialized staff, as the simulations have been precomputed, allowing for an estimate of a safe concentration. Moreover, the number of drugs used to depict the ROC curves is significantly higher than the number of compounds used in most previous works.

The new Tx index has significantly reduced the misclassification obtained with hERG test, although some false positive and false negative predictions with hERG tests are also misclassified with our Tx index. There are many drug-related aspects that were not included in our work mainly due to information unavailability, such as trafficking inhibition, the effects on other pathways, the presence of metabolites, and higher levels of the compound in the myocardium than in blood plasma. Although these aspects can also apply to properly classified drugs, they could have favored the misclassification of some drugs. For example, it has been reported that donepezil prolongs QT interval not only by $I_{\mathrm{Kr}}$ block but also by $I_{\mathrm{Kr}}$ trafficking inhibition. ${ }^{40}$ Additionally, QT prolongation by dasatinib is also partially due to its effects on intracellular PI3K signaling. ${ }^{41}$ Cilostazol is a phosphodiesterase 3 (PED3) inhibitor that induces intracellular cAMP elevation, $\mathrm{Ca}^{2+}$ dynamic unbalance, and early after depolarizations (EADs), ${ }^{42}$ which could favor TdP. Dronedarone is known to transform into $\mathrm{N}$-debutyl-dronedarone, ${ }^{43}$ a metabolite that retains up to one-third of the parent's activity. Because both show important channel blocking effects, not considering dronedarone's main metabolite might be the cause of its misclassification. As for saquinavir, it could be related to the presence of higher levels in myocardium than in blood plasma, ${ }^{44}$ increasing the odds of producing adverse effects in this tissue. Finally, other factors can be related to the misclassification of ofloxacin, quetiapine, ranolazine, lamotrigine, and eltrombopag. Although ofloxacin has been classified as a class 2 compound by CredibleMeds, rare cases of $\mathrm{TdP}$ have been reported in patients receiving it $^{45,46}$ and experimental data has shown that ofloxacin does not provoke APD prolongation in rabbit Purkinje fibers, ${ }^{47}$ which are cells prone to APD prolongation. In our work, we performed a large number of simulations to take into account the block effect if the drugs on the three main channels $\left(I_{\mathrm{Kr}}, I_{\mathrm{Ks}}\right.$, and $I_{\mathrm{CaL}}$ ), but a few drugs in our data set show blocking effects on the $\mathrm{Na}^{+}$channels, namely quetiapine, ranolazine, and lamotrigine. Finally, it is worth noting that eltrombopag showed high variability of its $\mathrm{IC}_{50}$, and its classification was uncertain as there is a lack of strong evidence for placing it in any QT risk category. $^{31}$

This study is also in line with current initiatives aiming to define a new paradigm in cardiac safety like comprehensive in vitro proarrhythmia assay $(\mathrm{CiPA}){ }^{22}$ where modeling and simulation of the human cellular ventricular electrophysiology will play a relevant role in the assessment of the proarrhythmic risk of drugs.

4.5. Limitations of the Study. One of the values of this work is the use of a large training series because we consider that this allows obtaining of a more realistic estimation of the prediction errors. However, the $I_{\mathrm{Ks}}$ and $I_{\mathrm{CaL}} \mathrm{IC}_{50}$ values of some compounds were not available from the literature, and it was assumed that the block of these channels was negligible, similarly to previous works. ${ }^{12,16}$ In addition, the $\mathrm{IC}_{50}$ values were obtained from heterogeneous sources, and it can be assumed that the experimental conditions were diverse. However, we avoided major sources of variability by selecting $\mathrm{IC}_{50}$ values registered in mammalian ventricular tissues when available and discarding those extracted from Xenopus oocytes (as described in the Methods section). We considered that all published data represents a distribution of values affected by random error plus variability due to differences in the experimental conditions. From this distribution, we picked the value at the center (the median), as a pragmatic approach to summarize all these values into a single one. Median is a robust estimator of the central tendency of a data sample, less sensitive to the presence of extreme values, and for this reason it was preferred over the average.

Our method does not include drug effects on $\mathrm{Na}^{+}$channels, which is related to the misclassification of quetiapine, ranolazine, and lamotrigine, which can significantly block $\mathrm{Na}^{+}$ channels at EFTPC. Future work will include this channel.

In our model, drug-channel interactions are simulated using the simple pore block model, which may be too simplistic. Consideration of the Hill coefficient of the sigmoidal curve fitted to the concentration-current response curves could provide little benefit, as suggested by other authors. ${ }^{15,16,31}$ Moreover, drug binding kinetics have not been considered in this paper although they have been related to increased susceptibility to acquired long QT syndrome in the presence of hERG channel kinetic abnormalities. ${ }^{26}$ Also, our simulations do not consider other aspects of the compounds, such as pharmacokinetics/pharmacodynamics, physiology impact, metabolites, or drug-drug interactions. It would have enriched the results of this study, unfortunately, there is a lack of experimental data for the inclusion of these phenomena for all the compounds considered in this study.

In this work, compounds have been classified using the clinical human torsadogenic risk classification from CredibleMeds, which feeds from clinical data. ${ }^{31}$ However, some of the compounds used in this study are classified differently in other sources, such as amiodarone, clarithromycin, clozapine, domperidone, donepezil, lapatinib, moxifloxacin, or ranolazine. $^{21}$ Indeed, a large number of compounds with contradicting classification have been listed by Wisniowska and Polak, ${ }^{21}$ who raise the need of a standardized classification for drug proarrhythmicity risk.

\section{CONCLUSIONS}

In this study, we introduce a new in silico tool to predict the effect of a drug on the APD and QT prolongation and its proarrhythmic risk using the valuable Tx index. It takes into account both the maximum EFTPC and the multichannel effect of the drugs. Although there is no doubt about the high influence of the hERG block of a drug on the QT prolongation and the likelihood of $\mathrm{TdP}$, our results highlight the need of considering the EFTPC and the blocking effects on the different channels of a drug for improving the prediction of cardiotoxicity, factors that are integrated in the Tx index. This work contributes to comprehensive in vitro proarrhythmia assay (CiPA) initiative by using an in silico human model to obtain a more accurate prediction of human QT and proarrhythmic risk of TdP. Our results show that all these four tests based on Tx provide very similar predictions and that good torsadogenic risk predictions can be obtained based on the endocardial APD prolongation $\left(\mathrm{Tx}-\mathrm{APD}_{\mathrm{Endo}}\right)$ of human isolated cells. This test could be used to predict the human QT prolongation caused by a compound in the different phases of 
drug discovery only using a test concentration and the blocking potency of the compound to $I_{\mathrm{Kr}}, I_{\mathrm{Ks}}$, and $I_{\mathrm{CaL}}$ or even to obtain an estimate of the maximum safe free plasma concentration that prevents excessive QT and APD prolongations.

\section{ASSOCIATED CONTENT}

\section{S Supporting Information}

The Supporting Information is available free of charge on the ACS Publications website at DOI: 10.1021/acs.jcim.7b00440.

Drug data set (risk class, EFTPC, $\mathrm{pIC}_{50}$, EFTPC calculation) with all references (PDF)

Simulation results (QT intervals, $\mathrm{APD}_{90}, \mathrm{Tx}-\mathrm{QT}$, and $\mathrm{Tx}-$ $\left.\mathrm{APD}_{90}\right)(\mathrm{PDF})$

Tx-APD ${ }_{\text {Endo }}$ ROC curves at $0.5,1$, and $2 \mathrm{~Hz}$ pacing rates (PDF)

\section{AUTHOR INFORMATION}

\section{Corresponding Author}

*Tel.: +34 963877000 ext. 76025. Fax: +34 96 3877093. Email: jsaiz@ci2b.upv.es.

ORCID $\odot$

Jordi Cano: 0000-0001-9461-9848

\section{Author Contributions}

${ }^{\S}$ Lucia Romero and Jordi Cano contributed equally to this work.

\section{Notes}

The authors declare no competing financial interest.

\section{ACKNOWLEDGMENTS}

L.R., J.C., J.G., B.T., J.S.: This work was partially supported by the Dirección General de Política Cientifica de la Generalitat Valenciana (PROMETEU2016/088) as well as Ministerio de Economía y Competitividad and Fondo Europeo de Desarrollo Regional (FEDER) DPI2015-69125-R (MINECO/FEDER, UE). F.S, M.P.: received support from the Innovative Medicines Initiative (IMI) Joint Undertaking under grant agreement no. 115002 (eTOX), resources of which are composed of financial contribution from the European Union's Seventh Framework Programme (FP7/2007-2013) and EFPIA companies' in-kind contributions.

\section{ABBREVIATIONS USED}

$\mathrm{APD}_{x}$, action potential duration at $x \%$ repolarization; CiPA, comprehensive in vitro proarrhythmia assay; $\mathrm{D}_{10}$, drug dose that produces a $10 \%$ increase in the $\mathrm{APD}_{90}$ of a single cell simulation or $\mathrm{QT}$ interval of the ECG; EAD, early after depolarization; ECG, electrocardiogram; EFTPC, effective free therapeutic plasma concentration (peak); hERG, ether-à-go-go related gene encoding the pore-forming $\alpha$-subunit of the rapid component of delayed rectifier current $\left(I_{\mathrm{Kr}}\right)$ channels in humans; $\mathrm{IC}_{50}$, half-maximal inhibitory concentration; $I_{\mathrm{CaL}}$, calcium type-L ion channel current; $I_{\mathrm{Kr}}$, rapid component of the delayed rectifier potassium current; $I_{\mathrm{Ks}}$, slow component of the delayed rectifier potassium current; ORd, O'Hara-Rudy model of the human ventricular cell ${ }^{30} ; \mathrm{pIC}_{50}$, negative logarithm of the $\mathrm{IC}_{50}$; $\mathrm{QT}$, time interval between the start of the $\mathrm{Q}$ wave and the end of the $\mathrm{T}$ wave in an ECG; ROC, receiver operating characteristic; TdP, torsade-de-pointes; $\mathrm{TNR}$, true negative rate; TPR, true positive rate; $\mathrm{XO}$, Xenopus oocytes

\section{REFERENCES}

(1) Hammond, T. G.; Carlsson, L.; Davis, A. S.; Lynch, W. G.; MacKenzie, I.; Redfern, W. S.; Sullivan, A. T.; Camm, A. J. Methods of Collecting and Evaluating Non-Clinical Cardiac Electrophysiology Data in the Pharmaceutical Industry: Results of an International Survey. Cardiovasc. Res. 2001, 49 (4), 741-750.

(2) Recanatini, M.; Poluzzi, E.; Masetti, M.; Cavalli, A.; De Ponti, F. QT Prolongation through hERG K+ Channel Blockade: Current Knowledge and Strategies for the Early Prediction during Drug Development. Med. Res. Rev. 2005, 25, 133-166.

(3) Noble, D. Computational Models of the Heart and Their Use in Assessing the Actions of Drugs. J. Pharmacol. Sci. (Tokyo, Jpn.) 2008, 107 (2), 107-117.

(4) Valentin, J.-P.; Hammond, T. Safety and Secondary Pharmacology: Successes, Threats, Challenges and Opportunities. J. Pharmacol. Toxicol. Methods 2008, 58 (2), 77-87.

(5) Adams, C. P.; Brantner, V. V. Estimating the Cost of New Drug Development: Is It Really 802 Million Dollars? Health Aff. 2006, 25 (2), 420-428.

(6) ICH Harmonised Tripartite Guideline. The Clinical Evaluation of QT/QTc Interval Prolongation and Proarrhythmic Potential for NonAntiarrhythmic Drugs E14, Step 4 Version; International Conference on Harmonisation of Technical Requirements for Registration of Pharmaceuticals for Human Use, May 2005; http://www.ich.org/ fileadmin/Public Web Site/ICH Products/Guidelines/Efficacy/ E14/E14 Guideline.pdf.

(7) E14 Clinical Evaluation of QT/QTc Interval Prolongation and Proarrhythmic Potential for Non-antiarrhythmic Drugs; U.S. Food and Drug Administration: Rockville, MD, October 2005; https://www.fda. gov/downloads/Drugs/GuidanceComplianceRegulatoryInformation/ Guidances/UCM073153.pdf.

(8) Roden, D. M. Cellular Basis of Drug-Induced Torsades de Pointes. Br. J. Pharmacol. 2008, 154 (7), 1502-1507.

(9) Kramer, J.; Obejero-Paz, C. A.; Myatt, G.; Kuryshev, Y. A.; Bruening-Wright, A.; Verducci, J. S.; Brown, A. M. MICE Models: Superior to the HERG Model in Predicting Torsade de Pointes. Sci. Rep. 2013, 3 (1), 2100.

(10) Windley, M. J.; Abi-Gerges, N.; Fermini, B.; Hancox, J. C.; Vandenberg, J. I.; Hill, A. P. Measuring Kinetics and Potency of hERG Block for CiPA. J. Pharmacol. Toxicol. Methods 2017, 88, 197.

(11) Pollard, C. E.; Abi Gerges, N.; Bridgland-Taylor, M. H.; Easter, A.; Hammond, T. G.; Valentin, J.-P. An Introduction to QT Interval Prolongation and Non-Clinical Approaches to Assessing and Reducing Risk. Br. J. Pharmacol. 2010, 159 (1), 12-21.

(12) Mirams, G. R.; Cui, Y.; Sher, A.; Fink, M.; Cooper, J.; Heath, B. M.; McMahon, N. C.; Gavaghan, D. J.; Noble, D. Simulation of Multiple Ion Channel Block Provides Improved Early Prediction of Compounds' Clinical Torsadogenic Risk. Cardiovasc. Res. 2011, 91 (1), 53-61.

(13) Obiol-Pardo, C.; Gomis-Tena, J.; Sanz, F.; Saiz, J.; Pastor, M. A Multiscale Simulation System for the Prediction of Drug-Induced Cardiotoxicity. J. Chem. Inf. Model. 2011, 51 (2), 483-492.

(14) Davies, M. R.; Mistry, H. B.; Hussein, L.; Pollard, C. E.; Valentin, J.-P.; Swinton, J.; Abi-Gerges, N. An in Silico Canine Cardiac Midmyocardial Action Potential Duration Model as a Tool for Early Drug Safety Assessment. Am. J. Physiol. Heart Circ. Physiol. 2012, 302 (7), H1466-H1480.

(15) Beattie, K. A.; Luscombe, C.; Williams, G.; Munoz-Muriedas, J.; Gavaghan, D. J.; Cui, Y.; Mirams, G. R. Evaluation of an in Silico Cardiac Safety Assay: Using Ion Channel Screening Data to Predict QT Interval Changes in the Rabbit Ventricular Wedge. J. Pharmacol. Toxicol. Methods 2013, 68 (1), 88-96.

(16) Mirams, G. R.; Davies, M. R.; Brough, S. J.; Bridgland-Taylor, M. H.; Cui, Y.; Gavaghan, D. J.; Abi-Gerges, N. Prediction of Thorough QT Study Results Using Action Potential Simulations Based on Ion Channel Screens. J. Pharmacol. Toxicol. Methods 2014, 70 (3), 246-254. 
(17) Yap, C. W.; Cai, C. Z.; Xue, Y.; Chen, Y. Z. Prediction of Torsade-Causing Potential of Drugs by Support Vector Machine Approach. Toxicol. Sci. 2004, 79 (1), 170-177.

(18) He, Y.; Lim, S. W. Y.; Yap, C. W. Determination of TorsadeCausing Potential of Drug Candidates Using One-Class Classification and Ensemble Modelling Approaches. Curr. Drug Saf. 2012, 7 (4), 298-308.

(19) Mistry, H. B.; Davies, M. R.; Di Veroli, G. Y. A New ClassifierBased Strategy for in-Silico Ion-Channel Cardiac Drug Safety Assessment. Front. Pharmacol. 2015, 6, 59.

(20) Lancaster, M. C.; Sobie, E. A. Improved Prediction of DrugInduced Torsades de Pointes Through Simulations of Dynamics and Machine Learning Algorithms. Clin. Pharmacol. Ther. 2016, 100 (4), 371-379.

(21) Wiśniowska, B.; Polak, S. Am I or Am I Not Proarrhythmic? Comparison of Various Classifications of Drug TdP Propensity. Drug Discovery Today 2017, 22 (1), 10-16.

(22) Sager, P. T.; Gintant, G.; Turner, J. R.; Pettit, S.; Stockbridge, N. Rechanneling the Cardiac Proarrhythmia Safety Paradigm: A Meeting Report from the Cardiac Safety Research Consortium. Am. Heart J. 2014, 167 (3), 292-300.

(23) Redfern, W. S.; Carlsson, L.; Davis, A. S.; Lynch, W. G.; MacKenzie, I.; Palethorpe, S.; Siegl, P. K. S.; Strang, I.; Sullivan, A. T.; Wallis, R.; Camm, A. J.; Hammond, T. G. Relationships between Preclinical Cardiac Electrophysiology, Clinical QT Interval Prolongation and Torsade de Pointes for a Broad Range of Drugs: Evidence for a Provisional Safety Margin in Drug Development. Cardiovasc. Res. 2003, 58 (1), 32-45.

(24) Gintant, G. An Evaluation of hERG Current Assay Performance: Translating Preclinical Safety Studies to Clinical QT Prolongation. Pharmacol. Ther. 2011, 129 (2), 109-119.

(25) Rodriguez, B.; Burrage, K.; Gavaghan, D.; Grau, V.; Kohl, P.; Noble, D. The Systems Biology Approach to Drug Development: Application to Toxicity Assessment of Cardiac Drugs. Clin. Pharmacol. Ther. 2010, 88 (1), 130-134.

(26) Romero, L.; Trenor, B.; Yang, P.-C.; Saiz, J.; Clancy, C. E. In Silico Screening of the Impact of hERG Channel Kinetic Abnormalities on Channel Block and Susceptibility to Acquired Long QT Syndrome. J. Mol. Cell. Cardiol. 2015, 87, 271-282.

(27) Trenor, B.; Gomis-Tena, J.; Cardona, K.; Romero, L.; Rajamani, S.; Belardinelli, L.; Giles, W. R.; Saiz, J. In Silico Assessment of Drug Safety in Human Heart Applied to Late Sodium Current Blockers. Channels 2013, 7 (4), 249-262.

(28) Okada, J. -i.; Yoshinaga, T.; Kurokawa, J.; Washio, T.; Furukawa, T.; Sawada, K.; Sugiura, S.; Hisada, T. Screening System for DrugInduced Arrhythmogenic Risk Combining a Patch Clamp and Heart Simulator. Sci. Adv. 2015, 1 (4), e1400142.

(29) Mirams, G. R.; Davies, M. R; Cui, Y.; Kohl, P.; Noble, D. Application of Cardiac Electrophysiology Simulations to proArrhythmic Safety Testing. Br. J. Pharmacol. 2012, 167 (5), 932-945.

(30) O’Hara, T.; Virág, L.; Varró, A.; Rudy, Y. Simulation of the Undiseased Human Cardiac Ventricular Action Potential: Model Formulation and Experimental Validation. PLoS Comput. Biol. 2011, 7 (5), e1002061.

(31) Woosley, R.; Romero, K. QTdrugs List; AZCERT, Inc.: 1822 Innovation Park Dr., Oro Valley, AZ 85755, 2013; www.Crediblemeds. org.

(32) Bottino, D.; Penland, R. C.; Stamps, A.; Traebert, M.; Dumotier, B.; Georgieva, A.; Helmlinger, G.; Lett, G. S. Preclinical Cardiac Safety Assessment of Pharmaceutical Compounds Using an Integrated Systems-Based Computer Model of the Heart. Prog. Biophys. Mol. Biol. 2006, 90 (1-3), 414-443.

(33) Brennan, T.; Fink, M.; Rodriguez, B. Multiscale Modelling of Drug-Induced Effects on Cardiac Electrophysiological Activity. Eur. J. Pharm. Sci. 2009, 36 (1), 62-77.

(34) Elkins, R. C.; Davies, M. R.; Brough, S. J.; Gavaghan, D. J.; Cui, Y.; Abi-Gerges, N.; Mirams, G. R. Variability in High-Throughput IonChannel Screening Data and Consequences for Cardiac Safety Assessment. J. Pharmacol. Toxicol. Methods 2013, 68 (1), 112-122.
(35) Hoffmann, P.; Warner, B. Are hERG Channel Inhibition and QT Interval Prolongation All There Is in Drug-Induced Torsadogenesis? A Review of Emerging Trends. J. Pharmacol. Toxicol. Methods 2006, 53 (2), 87-105.

(36) Cantilena, L., Jr.; Koerner, J.; Temple, R.; Throckmorton, D. FDA Evaluation of Cardiac Repolarization Data for 19 Drugs and Drug Candidates. Clin. Pharmacol. Ther. 2006, 79 (2), P29.

(37) Virág, L.; Iost, N.; Opincariu, M.; Szolnoky, J.; Szécsi, J.; Bogáts, G.; Szenohradszky, P.; Varró, A.; Papp, J. G. The Slow Component of the Delayed Rectifier Potassium Current in Undiseased Human Ventricular Myocytes. Cardiovasc. Res. 2001, 49 (4), 790-797.

(38) Romero, L.; Carbonell, B.; Trenor, B.; Rodríguez, B.; Saiz, J.; Ferrero, J. M. Systematic Characterization of the Ionic Basis of Rabbit Cellular Electrophysiology Using Two Ventricular Models. Prog. Biophys. Mol. Biol. 2011, 107 (1), 60-73.

(39) Zicha, S.; Moss, I.; Allen, B.; Varro, A.; Papp, J.; Dumaine, R.; Antzelevich, C.; Nattel, S. Molecular Basis of Species-Specific Expression of Repolarizing K+ Currents in the Heart. Am. J. Physiol. Heart Circ. Physiol. 2003, 285 (4), H1641-H1649.

(40) Cubeddu, L. X. Drug-Induced Inhibition and Trafficking Disruption of Ion Channels: Pathogenesis of QT Abnormalities and Drug-Induced Fatal Arrhythmias. Curr. Cardiol. Rev. 2016, 12 (2), 141-154.

(41) Damrongwatanasuk, R.; Fradley, M. G. Cardiovascular Complications of Targeted Therapies for Chronic Myeloid Leukemia. Curr. Treat. Options Cardiovasc. Med. 2017, 19 (4), 24.

(42) Kanlop, N.; Chattipakorn, S.; Chattipakorn, N. Effects of Cilostazol in the Heart. J. Cardiovasc. Med. 2011, 12 (2), 88-95.

(43) Iram, F.; Ali, S.; Ahmad, A.; Khan, S. A.; Husain, A. A Review on Dronedarone: Pharmacological, Pharmacodynamic and Pharmacokinetic Profile. J. Acute Dis. 2016, 5 (2), 102-108.

(44) Zhang, X.; Jordan, P.; Cristea, L.; Salgo, M.; Farha, R.; Kolis, S.; Lee, L. S. Thorough QT/QTc Study of Ritonavir-Boosted Saquinavir Following Multiple-Dose Administration of Therapeutic and Supratherapeutic Doses in Healthy Participants. J. Clin. Pharmacol. 2012, 52 (4), 520-529.

(45) Poluzzi, E.; Raschi, E.; Motola, D.; Moretti, U.; De Ponti, F. Antimicrobials and the Risk of Torsades de Pointes: The Contribution from Data Mining of the US FDA Adverse Event Reporting System. Drug Saf. 2010, 33 (4), 303-314.

(46) Briasoulis, A.; Agarwal, V.; Pierce, W. J. QT Prolongation and Torsade de Pointes Induced by Fluoroquinolones: Infrequent Side Effects from Commonly Used Medications. Cardiology 2011, 120 (2), $103-110$.

(47) Adamantidis, M. M.; Dumotier, B. M.; Caron, J. F.; Bordet, R. Sparfloxacin but Not Levofloxacin or Ofloxacin Prolongs Cardiac Repolarization in Rabbit Purkinje Fibers. Fundam. Clin. Pharmacol. 1998, 12 (1), 70-76.

(48) Briggs, K.; Cases, M.; Heard, D. J.; Pastor, M.; Pognan, F.; Sanz, F.; Schwab, C. H.; Steger-Hartmann, T.; Sutter, A.; Watson, D. K.; Wichard, J. D. Inroads to Predict in Vivo Toxicology-An Introduction to the eTOX Project. Int. J. Mol. Sci. 2012, 13, 38203846.

(49) Sanz, F.; Carrió, P.; López, O.; Capoferri, L.; Kooi, D. P.; Vermeulen, N. P. E.; Geerke, D. P.; Montanari, F.; Ecker, G. F.; Schwab, C. H.; Kleinöder, T.; Magdziarz, T.; Pastor, M. Integrative Modeling Strategies for Predicting Drug Toxicities at the eTOX Project. Mol. Inf. 2015, 34, 477-484. 